\title{
Demanda de productos animales sostenibles: leche de cabra de pastoreo
}

\author{
Ureña, L.P. ${ }^{\circledR}$; Colombo, S. '; López, A.L. ${ }^{2}$ y Ruiz, FA?
}

'Área de Economía de la cadena alimentaria; IFAPA Centro Camino de Purchil. Granada. España.

${ }^{2}$ Área de Ingeniería y Tecnología Agroalimentaria; IFAPA Centro Hinojosa del Duque. Córdoba. España.

\section{PALABRAS CLAVE}

Análisis de clúster.

Análisis de texto.

Comercialización.

Consumidores.

Innovación.

\section{RESUMEN}

La leche de pastoreo es una certificación privada que se están implantando en el mercado europeo, principalmente para leche fresca y queso de vaca, debido a su asociación con atributos de salud, calidad y sostenibilidad. La leche de cabra de pastoreo, por sus particularidades productivas y ecosistémicas, puede ser una alternativa de mercado. En el presente estudio, se diseñó una encuesta online para una muestra representativa de consumidores $(n=423)$, y mediante un estudio de clúster se segmentó en base a: i) ocho afirmaciones de hábitos de compra y ii) siete afirmaciones sobre la leche de pastoreo de cabra. Los consumidores se clasificaron en tres clusters: i) COMPROMETIDOS CALIDAD Y PASTOREO ( $n=210)$, ii) PASTORALES ( $n=139$ ) y iii) COMPROMETIDOS PERO INDECISOS $(n=70)$. Posteriormente, se realizó un análisis mixło de texto, usando tres palabras de asociación por consumidor con respecto a la leche de cabra de pastoreo ( $n=1072$ ). Los resultados indican que los consumidores valoran positivamente la leche de pastoreo de cabra, especialmente aquellos en el clúster $\mathrm{A}$ : son de mayor edad, y tendrían una mayor disposición a pagar este producto, asociándola a ideas como la salud, la protección del medioambiente y la calidad sensorial. Este estudio pretende sondear las posibilidades de la leche de cabra y sus productos para crear herramientas que optimicen su futura comercialización.

\section{Niche markets for sustainable animal products: pasture-raised goat milk.}

\section{SUMMARY}

\section{ADDITIONAL KEYWORDS}

Cluster analysis.

Consumers.

Innovation.

Marketing.

Text analysis.

\section{INFORMATION}

Cronología del artículo.

Recibido/Received: 08.05.2020

Aceptado/Accepted: 23.07.2020

On-line: 15/01/2021

Correspondencia a los autores/Contact e-mail:

luisp.urena@juntadeandalucia.es
Pastureraised milk is a private label which is being developed more and more in the European market, mainly for raw cow's milk and cheese, because of its association with health, quality and sustainability attributes. Due to its productive and ecosistemics particularities, pastureraised goat milk could be an alternative market option. In the present study a representative sample $(n=423)$ of Spanish consumers was interviewed through online survey. The sample was then segmented using a cluster analysis based on: i) eight personal statements about shopping and ii) seven assesments about pastureraised goat milk. Consumers were classified into three cluster: i) QUALTY AND PASTURE CONSCIUS ( $n=210)$, ii) PASTORAL ( $n=139$ ) y iii) CONSCIUS IN DOUBT ( $n=70)$. Subsequently, a mixed text analysis was made using three association words per consumer with pastureraised goat milk ( $n=1072)$. Results show that consumers highly appreciate pastureraised goat milk, specially population within cluster $A$ : these consumers are older, and they would have a higher willingness to pay for it, linked with perceptions such as health, environmental protection and sensory quality. This study aims to explore marketing possibilities of goat milk and derivated products, in order to create tools to optimize its future marketing.

\section{INTRODUCCIÓN}

Para el consumidor, la preocupación por acceder a productos que den respuesta a un desarrollo sostenible es cada vez mayor, y por ello debe ser una de las estrategias clave de cara a la diferenciación de los productos en el mercado (Mena et al., 2014a; Valdivielso et al., 2016). Así, además de la producción ecológica, la industria agroalimentaria ha ido articulando una respuesta mediante el uso de certificaciones, que ga- rantizan las condiciones sostenibles de producción de alimentos (Sepúlveda et al., 2013; Rubino, 2014).

La producción ecológica, normalizada en Europa principalmente a través del Reglamento (CE) 834/2007 del Consejo (28 de junio de 2007) sobre producción y etiquetado de los productos ecológicos, desarrollado por los Reglamento (CE) 889/2008 de 5 de septiembre de 2008 y el Reglamento (CE) 1235/2008 de la Comisión, es uno de estos modelos de garantía certificada, quizás el más representativo en la actualidad. Europa 
contaba en 2017 con una superficie certificada como ecológica de 14.6 millones de hectáreas, con España en primer lugar con 2 millones de hectáreas (FiBL e IFOAM, 2017). Desde 2008 a 2017, el mercado ecológico europeo dobló su volumen de producción, creciendo en el último año un 10,5\% en Europa (Willer et al., 2019). En cuanto a la leche, 4,7 millones de toneladas métricas de leche de vaca ecológica fueron producidas en 2017 (Willer et al., 2019), suponiendo el 3\% de la leche total europea.

Además de la producción ecológica están apareciendo otras iniciativas privadas donde se utilizan atributos ligados a la sostenibilidad para la comercialización y la defensa de la ganadería (Niles, 2013; Mena et al., 2014b; Grunert et al., 2014), así como también modelos más concretamente ligados a la industria láctea (Djekic et al., 2018). Ejemplos de este reciente aumento son las distintas certificaciones sobre el bienestar animal (Lundmark et al., 2018), bien sea de la leche de pastoreo (Montejano, 2018) y su adopción por parte de grandes cadenas de distribución (Méndez, 2018), así como para otros productos de origen animal como la carne (Van Loo et al., 2014) o los huevos (Gerini et al., 2016).

En cuanto a la leche de cabra, Europa representa el 16,6\% de la producción mundial (FAOSTAT, 2018), siendo España el segundo productor de Europa (14,1\%; 328 litros/animal) (Pulina et al., 218) y, junto a Francia y Grecia, agrupa el 70\% del censo caprino lechero y más del 80\% de la producción de leche de cabra europea (MAPA, 2018).

Andalucía es la región que contabiliza el mayor número de rebaños caprinos lecheros en 2018 (3491, el $52 \%$ del total en España) (MAPA, 2018), con un tamaño de explotación generalmente pequeño (tamaño medio de 64 animales) pero heterogéneo, si tenemos en cuenta que un tercio de las explotaciones producen el $87 \%$ de la leche de cabra (Pulina et al., 2018). Esta heterogeneidad es común denominador en la mayoría de países europeos, y se refleja en sus distintos sistemas de producción y razas autóctonas (Gutiérrez, 2018a, Ruiz et al., 2019), con multitud de sistemas de gestión pastorales: pastoreo continuo, estacional, trashumancia, etc.

A pesar de que productos como la leche de cabra de pastoreo pueden llegar a satisfacer las demandas de los consumidores respecto a una producción ética y sostenible de alimentos, siendo menos vulnerables a los cambios del mercado (Bernués et al., 2011), y una herramienta para que éstos modelos se mantengan en el tiempo (Ruiz et al., 2019), actualmente no existe oferta de este tipo de producto en el mercado. El objetivo de este estudio es caracterizar y categorizar la actitud del consumidor sobre los atributos que ofrece la leche de cabra de pastoreo en la segunda región productora de leche de cabra de la Unión Europea.

\section{MATERIAL Y MÉTODOS}

\section{DISEÑO Y RECOLECCIÓN DE DATOS}

Se diseñó una encuesta online basada en otros estudios de mercado con consumidores (Newberry e Israel, 2017). Para ello se utilizó la herramienta Google Forms
(Alphabet Co., Mountain View, CA) de la plataforma docs.google (Google, 2019) por su potencialidad y accesibilidad multiplataforma (Anderson, 2019).

El cuestionario consta de 33 ítems divididos en 7 apartados: i) datos sociodemográficos (edad, sexo, nivel de estudios y lugar de residencia); ii) hábitos de salud (fumador/a o no, alergias/intolerancias alimentarias); iii) hábitos de consumo de productos lácteos (frecuencia consumo leche, especie animal, consumo bebida vegetal, tipo de leche consumida, lugar y razón de compra de leche y conocimiento general de certificaciones); iv) hábitos de compra (establecimiento habitual de compra, razón principal de compra, certificaciones que conoce de leche y pregunta de control); ; v) categorizar ocho afirmaciones sobre sus hábitos de compra y consumo sostenibles; vi) categorizar siete afirmaciones sobre los atributos sostenibles de la leche de pastoreo; y vii) breve explicación muy genérica sobre la leche de pastoreo, se pregunta acerca de las tres primeras palabras o conceptos que le vinieran a la mente sobre leche de pastoreo.

Todas las cuestiones se ordenaron de forma aleatoria, buscando evitar los efectos del orden en las respuestas (Schuman y Presser, 1981). Se usó una escala de Likert de 5 puntos, compuesta desde el punto 1 ("Totalmente en desacuerdo") hasta el punto 5 ("Totalmente de acuerdo) para la categorización de las afirmaciones de hábitos de compra y consumo (apartado vi) del cuestionario), y las de leche de pastoreo (apartado vii del cuestionario). La escala de 5 puntos, debido a la difusión telemática de la encuesta, fue elegida en busca de la mayor simplificación para el encuestado (Joshi et al., 2015).

La población total objeto de estudio fue la región de Andalucía, que según los últimos censos estadísticos es de 8384408 personas (Instituto de Estadística y Cartografía de Andalucía, 2019), y que por tanto a efectos estadísticos se puede considerar como una población infinita. Así, para asegurar la representatividad de la muestra con respecto a la población andaluza (AriasGómez et al., 2016), se realizó un muestreo aleatorio proporcional estratificado, estableciendo cuotas máximas de respuestas en cuanto las variables demográficas de sexo, provincia de residencia y nivel de estudios (Instituto de Estadística y Cartografía de Andalucía, 2019), asumiendo un nivel de confianza del 95.5\% y una precisión del muestreo del $\pm 5 \%$ hasta llegar a una muestra mínima de 400 respuestas válidas.

La toma de datos se realizó entre el 27 de febrero y el 15 de marzo de 2020. Se establecieron como criterios de exclusión (Arias-Gómez et al., 2016) los siguientes supuestos: ser menor de edad, tener residencia fuera de Andalucía y no consumir leche. Además, a lo largo del cuestionario se incluyó una pregunta de control (apartado 4), con el fin de eliminar los participantes que no prestaran la suficiente atención.

Se obtuvo un total de 457 respuestas, de éstas eliminaron las que cumplían con los criterios de exclusión, así como las que respondían erróneamente a la pregunta de control. También, se identificaron como "straigth-liners" (encuestados que siempre contestan el mismo valor) aquellos con menor nivel de formación 
y mayor rapidez de respuesta (Zhang y Conrad, 2014). Finalmente se analizaron 423 respuestas correctas, cuya ficha técnica de muestreo se muestra en la Tabla I.

\section{ANÁlisis de Clústers}

Para la realización del análisis clúster se usaron las respuestas de las afirmaciones de hábitos de compra y consumo (apartado v del cuestionario), y las de leche de pastoreo (apartado vi del cuestionario), en relación con otros estudios similares en vacuno de leche (Weinrich et al., 2014; Kühl et al., 2017). El análisis jerárquico de clúster se realizó mediante el método de medida de las distancias euclídeas al cuadrado (Milligan y Hirtle, 2012) y la identificación de outliers por el método del vecino más cercano, donde se eliminaron cuatro respuestas más como outliers, quedando una muestra para el análisis clúster de 419 (n=419). Posteriormente, se usó el método de Ward y una representación en dendrograma para identificar y decidir los tres grupos resultantes.

Los clusters obtenidos se describieron en base a las características demográficas de los encuestados, sus hábitos de compra y consumo de leche, realizando para ello un análisis multivariante (Madry et al., 2013). A las variables cuantitativas se les cometió un análisis de las medias mediante un ANOVA de una vía, explorando la significancia $(\mathrm{P} \leq 0.05)$ de las diferencias entre clusters mediante la prueba post hoc de Tukey (para variables cuyas varianzas resultaron homogéneas según el test de Levene) o la prueba de Games-Howell, en caso de no homogeneidad. En el caso de las variables cualitativas se analizaron mediante test de Chi Cuadrado de Pearson, explorando las diferencias por las pruebas post hoc Phi y V de Cramer.

Para ello se utilizó el software estadístico IBM SPSS Statistics for Windows (IBM Corp. Released 2017. Version 25.0. Armonk, NY).

\section{Evaluación cualitativa: análisis de texto}

Tras una breve explicación acerca de lo que es la leche de pastoreo, acompañada de dos imágenes neutrales, y sin incluir información acerca de las ventajas o desventajas del modelo de producción, se requirió a los encuestados que anotaran las tres palabras que

Tabla I. Ficha técnica de muestro. Fuente: elaboración propia (Screen data sheet. Source: own elaboration).

\begin{tabular}{|c|c|}
\hline Universo & $\begin{array}{c}\text { Consumidores de leche > } 18 \text { años residentes } \\
\text { en Andalucía }\end{array}$ \\
\hline Ámbito & Andalucía \\
\hline $\begin{array}{l}\text { Tamaño mues- } \\
\text { tral }\end{array}$ & 423 \\
\hline Error muestral & $\pm 5 \%$ \\
\hline $\begin{array}{l}\text { Nivel de con- } \\
\text { fianza }\end{array}$ & $95.5 \%(\mathrm{k}=2)$ \\
\hline $\begin{array}{l}\text { Diseño de la } \\
\text { muestra }\end{array}$ & $\begin{array}{c}\text { Estratificado, cuotas máximas por: género, } \\
\text { provincia de residencia y nivel de estudios }\end{array}$ \\
\hline $\begin{array}{l}\text { Fecha de eje- } \\
\text { cución }\end{array}$ & Febrero - marzo 2020 \\
\hline
\end{tabular}

pensaran espontáneamente sobre la leche de pastoreo de cabra.

Se clasificaron las respuestas obtenidas en base a tres categorías principales: i) positivas, ii) negativas o iii) neutrales. Posteriormente, se reasignaron en subcategorías, matizando así su sentido de clasificación: i) Positivas: Salud, Bienestar animal y medioambiente, Sensorial, Generales, Modelo producción y Certificación; y ii) Negativas: Sensorial, Precio, Salud y Generales. Siguiendo la metodología de varios autores, resumida por Thomas (2006), dos expertos en diversificación y producción láctea, autores del presente trabajo, se encargaron de definir y posteriormente asignar las categorías y subcategorías, así como un tercer experto se encargó de dirimir los desacuerdos entre ambos . La codificación del texto y su análisis se realizó en combinación de técnicas de análisis deductivo e inductivo (Cuva, 2014). Debido al gran volumen de datos, para su codificación y análisis se empleó el software MaxQDA Analytics Pro 2020 (Provalis Research Released 2020. Version Freeware 20.0.7. Montreal, QC).

\section{RESULTADOS}

\section{DESCRIPCIÓN DE LA MUESTRA}

En la Tabla II aparecen los datos socio-demográficos obtenidos de la muestra con respecto a la población andaluza (Instituto de Estadística y Cartografía de Andalucía, 2019). Con respecto a la provincia de residencia la muestra no difiere de forma estadísticamente significativa, para un nivel de confianza del $90 \%\left(\chi^{2}=16.363\right.$; $\mathrm{p}=0.02)$, siendo con respecto al sexo distinta, $\left(\chi^{2}=\right.$ 13.492; $p=0.00)$. En la muestra hay un mayor número de mujeres, en línea con los estudios realizados acerca de una actitud de la mujer más positiva y concienciada como consumidora (Moosmayer y Fuljahn, 2010). Así mismo, la desviación en la formación con respecto a la población andaluza es debida a: i) el mayor acceso a herramientas de encuesta online por parte de personas con mayor formación (Díaz de Rada, 2012) y ii) el proceso de eliminación de los "straight-liners" (Zhang y Conrad, 2014).

\section{ClÚster}

Se realizó la agrupación de los consumidores en 3 segmentos o clúster, atendiendo a las menores distancias posibles (distancias euclídeas al cuadrado y el método de Ward) y tras la opción de agrupación en 5 segmentos. La Tabla III recoge los resultados de las variables "Afirmaciones" de cada uno de ellos junto a las variables socio-demográficas y de hábitos de consumo y compra de productos lácteos.

La descripción de los clusters es la siguiente:

- Clúster A COMPROMETIDOS CALIDAD Y PASTOREO, es el grupo más numeroso ( $n=210 ; 50.12 \%$ ), y de mayor edad. Los consumidores de este grupo se preocupan más por los aspectos medioambientales, y por la calidad de los productos agroalimentarios que adquieren, dándole importancia al proceso de compra. Valoran muy positivamente la leche de pastoreo y sus atributos de sostenibilidad. 
Tabla II. Descripción de la muestra $(n=423)(D e-$ scription of the sample $(n=423))$.

Ítem

\begin{tabular}{|c|c|c|}
\hline Ítem & $\begin{array}{c}\text { Muestra } \\
(\%)\end{array}$ & $\begin{array}{c}\text { Andalucía }{ }^{1} \\
(\%)\end{array}$ \\
\hline \multicolumn{3}{|l|}{ Sexo } \\
\hline Hombre & 40.66 & 49.30 \\
\hline Mujer & 59.34 & 50.70 \\
\hline Edad (años) & 44.86 & 41.56 \\
\hline \multicolumn{3}{|c|}{ Provincia residencia } \\
\hline Almería & 8.27 & 8.46 \\
\hline Cádiz & 13.48 & 14.77 \\
\hline Córdoba & 12.77 & 9.37 \\
\hline Granada & 13.71 & 10.88 \\
\hline Huelva & 7.09 & 6.20 \\
\hline Jaén & 9.22 & 7.61 \\
\hline Málaga & 14.66 & 19.57 \\
\hline Sevilla & 20.80 & 23.14 \\
\hline \multicolumn{3}{|l|}{ Estudios } \\
\hline Sin estudios & 0.24 & 9.21 \\
\hline Primarios & 5.44 & 13.19 \\
\hline $\begin{array}{l}\text { Secunda- } \\
\text { rios }\end{array}$ & 14.18 & 52.74 \\
\hline Superiores & 80.14 & 24.86 \\
\hline
\end{tabular}

${ }^{1}$ Instituto de Estadística y Cartografía de Andalucía (2018).

- Clúster B: PASTORALES, es el segundo grupo ( $\mathrm{n}=$ 139; $33.17 \%$ ) En los consumidores de este clúster, dan menos importancia a los aspectos medioambientales, que el producto esté certificado y al proceso de compra. Aunque tienen una posición menos preocupada en la compra, valoran bastante positivamente los atributos que produce la leche de pastoreo.

- Clúster C: COMPROMETIDOS PERO INDECISOS, es el grupo menos numeroso ( $n=70 ; 16.70 \%)$ agrupan sus respuestas en una situación intermedia al resto de clúster para todas las afirmaciones de hábitos de consumo y compra, pero son los que ofrecen las puntuaciones más bajas en la valoración de los atributos ligados al pastoreo, a pesar de ello los valoran positivamente.

\section{iii. Análisis de texto}

Todos los encuestados contestaron a las tres preguntas abiertas al menos con una palabra, contabilizándose un total de 1300 palabras. Se obviaron las respuestas sin relación con el objeto (leche de pastoreo de cabra), se eliminaron todas las palabras de menos de 4 letras, así como aquellas cuya frecuencia fue menor de 3 veces. Finalmente, un total de $1072(n=1072)$ palabras fueron recogidas para su análisis. No se encontraron diferencias estadísticamente significativas entre los clusters en referencia a las palabras utilizadas.
Al clasificarlas se obtuvieron 955 afirmaciones positivas $(89.09 \%$; $=1072), 52$ negativas $(4.85 \%)$ y 65 neutrales $(6.06 \%)$. Explorando más de cerca los resultados, se observa cómo el grupo principal de respuestas se ha articulado alrededor de la subcategoría Salud $(20.06 \%)$, incluyendo palabras como "natural", "salud", "sana", "saludable" y "nutritiva"; seguida de la subcategoría Bienestar animal y medioambiente (17.82 $\%$ ), con "sostenibilidad", "bienestar animal", "medio ambiente", "ecología", "ecosistema", "biodiversidad" y "verde", y Sensorial (17.72\%) con términos como "sabor", "calidad", "fresca" y "sabrosa". Posteriormente, se ha caracterizado el subapartado Generales (15.39\%) englobando conceptos poco concretos como "infancia", "alternativa", "mercado", etc.

Por el contrario, la principal categoría de carácter negativo ha sido la Sensorial (16.70 \%) conformada por palabras como "fuerte", "desagradable y "densa", seguida de lejos por Precio (1.49\%) con la palabra "cara" como principal componente.

En la categoría Neutrales se han clasificado aquellas palabras que, por falta de contexto o por ser conceptos generales, no pueden clasificarse con un cariz positivo o negativo, tales como "queso", "cabra", "leche" o "desconocimiento".

A continuación, se presentan las frecuencias obtenidas (Tabla IV y Figura 1) así como su clasificación en categorías y subcategorías que explican mejor el sentido positivo, neutro o negativo (Tabla V).

\section{DISCUSIÓN}

\section{Oferta de leche de cabra de pastoreo}

La ganadería caprina de leche, a diferencia de lo ocurrido con el vacuno, sigue manteniendo sistemas tradicionales de producción basados en el pastoreo (Ruiz et al., 2019). Actualmente los mayores productores europeos conservan modelos donde el pastoreo forma parte fundamental del manejo alimentario: Andalucía en España (ejemplo de la raza Payoya), la Provenza en el sur Francia (modelo "fermiers"), Cerdeña en Italia (raza Sarda) o Grecia (modelo de pequeñas granjas o "perivoli") (Ruiz et al., 2009; Pazzola et al., 2017; Ntassiou y Doukas, 2019). Esta imbricación del territorio y de los modelos productivos tradicionales del caprino puede explicar el por qué los consumidores tiene una alta percepción de los productos procedentes del pastoreo, y concretamente en lo que se refiere a la leche de cabra de pastoreo en este estudio, a pesar de ser un producto aún por implantar en los establecimientos, la asocian efectivamente con términos positivos como "infancia", "natural" o "sostenibilidad", así como mayoritariamente la consideran un producto alineado con sus exigencias en cuanto a atributos de sostenibilidad.

La leche ecológica es la única figura legislativa oficial donde el pastoreo aparece de forma explícita como requisito legal. Sin embargo, el proceso de conversión y la gestión posterior de este modelo de producción, la cantidad y calidad de los insumos, sobre todo piensos y forrajes, y la falta de una línea clara de comercialización, entre otras, han constituido un freno para el 


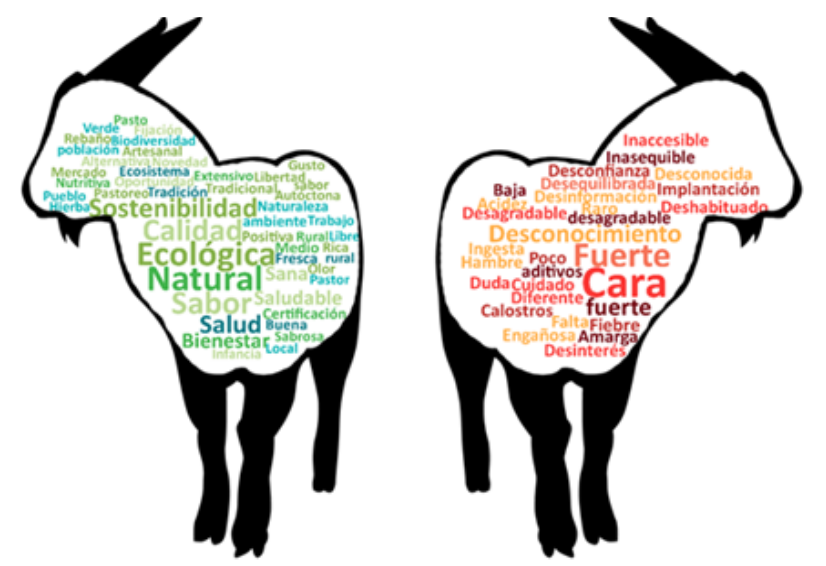

Figura 1. Palabras de asociación positivas (izquierda y negativas (derecha de los encuestados con respecto a la leche de cabra de pastoreo $(n=1072$; a mayor frecuencia, mayor tamaño letra) (Positive association words (left and negative (right of respondents with respect to grazing goat's milk ( $n=1072$; The larger the letter size the higher the frequency).

pleno desarrollo del sector caprino pastoral europeo hacia este modelo (Castel et al., 2010; Ruiz et al., 2019).

Pero quizás el reto más importante es el de la propia definición de leche de pastoreo. Los términos "criado en pastos", "pastoreado en prados", "terminado en pasto" o "alimentado con pasto", al referirse a la leche o la carne, no están definidos a nivel legislativo, ni en los EE.UU. ni en la Unión Europea, donde se han realizado la mayoría de los estudios (Stampa et al., 2020), y puede explicar los resultados de este estudio, donde los consumidores asocian a la leche de cabra de pastoreo palabras como "desconocimiento". Esto es fundamental para poder seguir avanzando en el proceso de puesta en valor de esta leche, donde ya las empresas lácteas y distribuidoras ven una oportunidad de mercado (Hennesy et al., 2020).

\section{HÁBITOS SOSTENIBLES DE CONSUMO Y COMPRA}

Tradicionalmente se ha considerado que los factores sociodemográficos como la edad y el sexo tienen un impacto limitado en el consumo de productos certificados y productos ecológicos (Nayga, 1997; Thompson, 1998), si bien estudios más recientes expresan que tanto la edad como el género son factores de varianza, manteniendo la mujer una actitud más positiva y concienciada como consumidora (Lea y Worsley, 2005; Moosmayer y Fuljahn, 2010). En el presente estudio, se han recogido una mayoría de respuestas de mujeres $(59.34 \%)$, aunque no se han encontrado diferencias significativas entre los clusters, existiendo las mismas proporciones en todos los grupos definidos.

También la formación/estudios de los encuestados puede ser un factor a tener en cuenta; algunos estudios relacionan el nivel de estudios con los hábitos de consumo de los consumidores, asociando la formación superior/universitaria a una mayor preocupación por la calidad y salubridad de los productos que consumen (Kraus et al., 2017). En nuestro caso concreto, la desviación en la formación superior con respecto a la población andaluza se debe, de una parte, por el mayor acceso a herramientas de encuesta online por parte de personas con mayor formación (Díaz de Rada, 2012), así como por el proceso de eliminación de los "straightliners" (Zhang y Conrad, 2014).

Existen diferencias estadísticamente significativas para el análisis de clusters con respecto a la edad, siendo más elevada la del grupo A (47.45), con respecto al B (42.16) y el C (42.13), al igual que en otras investigaciones de consumo de alimentos (Mogendi et al., 2016; Kraus et al., 2017), donde los consumidores de mayor edad, principalmente mujeres, son los más preocupados por las características intrínsecas de su alimentación como la calidad nutricional, la seguridad alimentaria y la calidad sensorial.

En cuanto a los resultados obtenidos para las preguntas sobre hábitos de consumo y compra de lácteos no existen diferencias entre clusters. Los encuestados en este estudio declaran consumir normalmente leche varias veces al día, fundamentalmente de vaca y semidesnatada, realizando la compra en supermercado (Tabla III). Estos datos están en línea con los recogidos para el conjunto de España por la Organización Interprofesional Láctea (InLac) (Bustillo, 2015). La razón principal de compra de leche por los consumidores es la Salud (36 \%). Otros autores apuntan al hecho de que los consumidores españoles valoran muy positivamente la presencia de una certificación tanto de calidad como de producción ecológica (Scarpato et al., 2017), y que esto en gran medida se ve aumentado en consumidores preocupados de forma global respecto a la salud. Estas afirmaciones pueden explicar los resultados más altos del clúster A de este estudio, con respecto a las preguntas sobre hábitos sostenibles de compra y consumo: Pregunta 4 "Pago-certificados ecológicos/biológicos" y Pregunta 8 "Compra productos alimenticios de calidad".

En la misma línea, otros estudios (Vanhonacker et al., 2007; Gillespiex et al., 2013; Vanhonacker y Verbeke, 2014) indica una tendencia actual de aumento de la preocupación de los consumidores respecto al bienestar animal y el medioambiente, aunque también respecto a la certificación de éstos y otros extremos en la producción animal (Heerwagen et al., 2015). Los resultados expresan opiniones de los consumidores en la misma dirección: Pregunta 1 "El bienestar animal es importante para mí", Pregunta 2 "El medioambiente y su protección son importantes para mí", Pregunta 3 "Pago de marcas reconocidas" y Pregunta 4 "Pago-certificados ecológicos/biológicos" se encontraron puntuaciones elevadas y además diferencias significativas del clúster A (el de mayor número de consumidores) con el resto de grupos.

En cambio, es el grupo o clúster $\mathrm{C}$ el que registra las puntuaciones más bajas al respecto de las mismas cuestiones Esto puede ser debido a una falta de conciencia medioambiental que, tal y como marcan otras investigaciones, tiene relación con la confianza en la marca y certificados, y por tanto desde este punto de vista se entiende la importancia de su mejora a través de herramientas como la información al consumidor o la limitación del ruido informativo en el mercado (Testa et al., 2015; Wei et al., 2017). 


\section{AtributOS DEL CAPRINO DE PASTOREO}

La incorporación del acceso a los pastos en los sistemas de producción lechera reporta considerables beneficios para el bienestar de los animales (Arnott et al. 2017; Charlton y Rutter, 2017). De forma estadísticamente significativa, tal y como se observa en la Tabla III y a un nivel de significación de 0.05 , la afirmación "La leche de pastoreo implica un alto bienestar animal" es más valorada por el Clúster $\mathrm{A}$, seguida del Clúster $\mathrm{B}$ y del C. En un estudio realizado con consumidores alemanes, se muestra que el grupo con mayor interés sobre el bienestar animal es el que se informa más (Heise y Theuvsen, 2016), en concordancia con lo obtenido en este trabajo para el Clúster A. El interés del bienestar animal como motivo positivo de compra ha sido estudiado en varios trabajos (Ingenbleek y Immink, 2011; Van Riemsdijk et al., 2017); por tanto, que se considere que el pastoreo implica un alto bienestar animal es muy positivo para su comercialización.

Las prácticas de pastoreo controlado desempeñan un papel eficaz en la limpieza de los combustibles de superficie (plantas herbáceas arbustos y otros com-

Tabla III. Análisis de clusters, variables socio-demográficas y hábitos de consumo y compra de lácteos ( $\mathrm{n}=$ 419) (Analysis of clusters, socio-demographic variables and consumption and dairy purchasing habits $(n=419)$ ).

\begin{tabular}{|c|c|c|c|c|}
\hline Clúster & & Clúster $\mathrm{A}^{2}$ & Clúster $\mathrm{B}^{2}$ & Clúster $\mathrm{C}^{2}$ \\
\hline Tamaño & & 210 & 139 & 70 \\
\hline Afirmación ${ }^{1}$ & F-valor & & & \\
\hline 1.El bienestar animal es importante para mí) & 66.08 & $4.75^{\mathrm{bc}} \pm 0.03$ & $3.86^{\mathrm{a}} \pm 0.07$ & $4.23^{\mathrm{a}} \pm 0.10$ \\
\hline 2.El medioambiente y su protección son importantes para mí & 64.85 & $4.86^{\mathrm{bc}} \pm 0.03$ & $4.14^{\mathrm{ac}} \pm 0.06$ & $4.53^{\mathrm{ab}} \pm 0.07$ \\
\hline $\begin{array}{l}\text { 3.No me importa pagar un poco más por productos de marca } \\
\text { reconocidas }\end{array}$ & 69.81 & $4.20^{\mathrm{bc}} \pm 0.06$ & $3.07^{\mathrm{ac}} \pm 0.07$ & $3.77^{\mathrm{ab}} \pm 0.10$ \\
\hline $\begin{array}{l}\text { 4.No me importa pagar un poco más por productos certificados } \\
\text { como ecológicos/biológicos }\end{array}$ & 125.78 & $4.41^{\mathrm{b}} \pm 0.05$ & $3.07^{\mathrm{ac}} \pm 0.08$ & $4.21^{\mathrm{b}} \pm 0.09$ \\
\hline 5.Primero de todo me fijo en el precio cuando compro comida & 12.24 & $2.70^{\mathrm{b}} \pm 0.08$ & $3.11^{\mathrm{ac}} \pm 0.08$ & $2.37^{\mathrm{b}} \pm 0.13$ \\
\hline 6.Para mí, comprar comida debe ser rápido & 9.18 & $2.68^{b} \pm 0.08$ & $3.19^{\mathrm{a}} \pm 0.09$ & $2.71^{\mathrm{b}} \pm 0.06$ \\
\hline $\begin{array}{l}\text { 7.Me tomo mi tiempo para informarme bien sobre la comida } \\
\text { que compro }\end{array}$ & 40.11 & $4.11^{\mathrm{b}} \pm 0.05$ & $3.32^{\mathrm{ac}} \pm 0.07$ & $3.96^{b} \pm 0.10$ \\
\hline $\begin{array}{l}\text { 8.Me describiría a mí mismo/a como muy preocupado con la } \\
\text { calidad de la comida que compro }\end{array}$ & 64.47 & $4.44^{\mathrm{bc}} \pm 0.04$ & $3.55^{\mathrm{ac}} \pm 0.07$ & $4.10^{\mathrm{ab}} \pm 0.10$ \\
\hline 1P.La leche de pastoreo implica un alto bienestar animal & 83.42 & $4.77^{\mathrm{bc}} \pm 0.04$ & $4.27^{\mathrm{ac}} \pm 0.06$ & $3.64^{\mathrm{ab}} \pm 0.10$ \\
\hline 2P.La leche de pastoreo previene la aparición de incendios & 151.82 & $4.85^{\mathrm{bc}} \pm 0.03$ & $4.30^{\mathrm{ac}} \pm 0.07$ & $3.21^{\mathrm{ab}} \pm 0.12$ \\
\hline $\begin{array}{l}\text { 3P.La leche de pastoreo tiene una alta calidad sensorial y } \\
\text { nutricional }\end{array}$ & 80.94 & $4.68^{\mathrm{bc}} \pm 0.06$ & $4.04^{\mathrm{ac}} \pm 0.09$ & $3.64^{\mathrm{ab}} \pm 0.04$ \\
\hline $\begin{array}{l}\text { 4P.La leche de pastoreo conlleva que los animales salgan a } \\
\text { campo todos los días }\end{array}$ & 81.43 & $4.83^{\mathrm{bc}} \pm 0.03$ & $4.50^{\mathrm{ac}} \pm 0.05$ & $3.81^{\mathrm{ab}} \pm 0.10$ \\
\hline $\begin{array}{l}\text { 5P.La leche de pastoreo favorece la fijación de la población } \\
\text { en el medio rural }\end{array}$ & 95.78 & $4.87^{\mathrm{bc}} \pm 0.03$ & $4.40^{\mathrm{ac}} \pm 0.06$ & $3.77^{\mathrm{ab}} \pm 0.09$ \\
\hline $\begin{array}{l}\text { 6P.La leche de pastoreo promueve la defensa del medio } \\
\text { ambiente }\end{array}$ & 195.15 & $4.87^{\mathrm{bc}} \pm 0.02$ & $4.46^{\mathrm{ac}} \pm 0.04$ & $3.43^{\mathrm{ab}} \pm 0.10$ \\
\hline $\begin{array}{l}\text { 7P.La leche de pastoreo implica el uso de razas ganaderas } \\
\text { autóctonas }\end{array}$ & 86.27 & $4.63^{\mathrm{bc}} \pm 0.04$ & $4.12^{\mathrm{ac}} \pm 0.07$ & $3.33^{\mathrm{ab}} \pm 0.10$ \\
\hline \multicolumn{5}{|c|}{ Atributos descriptivos } \\
\hline Edad media (años) & & $47.45^{\mathrm{bc}} \pm 0.80$ & $42.16^{\mathrm{a}} \pm 1.08$ & $42.13^{\mathrm{a}} \pm 1.41$ \\
\hline Fumador: No (\%) & & 81.43 & 82.73 & 80.00 \\
\hline Alergia/Intolerancia alimentaria: Sí (\%) & & 10 & 6.48 & 2.86 \\
\hline Frecuencia consumo leche: Varias veces al día (\%) & & 54.29 & 47.48 & 47.14 \\
\hline $\begin{array}{l}\text { Según la especie animal, tipo de leche más consumida: } \\
\text { Vaca (\%) }\end{array}$ & & 92.86 & 94.24 & 95.71 \\
\hline $\begin{array}{l}\text { Según el tipo, tipo de leche más consumida: Semidesnatada } \\
(\%)\end{array}$ & & 46.67 & 51.08 & 45.71 \\
\hline Dónde la compra: Supermercado (\%) & & 48.09 & 57.55 & 47.14 \\
\hline Razón principal de compra: Salud (\%) & & 40.48 & 28.78 & 40 \\
\hline
\end{tabular}

${ }^{1}$ los encuestados respondieron a las afirmaciones en base a una escala Likert de 5 puntos (1: totalmente en desacuerdo; 5: totalmente de acuerdo); ${ }^{2}$ los valores se muestran como media \pm error std; ${ }^{a-c}$ Distintas letras en la misma fila indican diferencias significativas $(p<0.05)$ en las pruebas post hoc. 
bustibles leñosos), si se practican en el momento adecuado, a nivel de existencias y en las zonas apropiadas (Robles et al., 2009). El pastoreo como herramienta en la prevención de incendio es la afirmación con mayor variación y diferente de forma significativa entre el Clúster A y el C, y se posiciona como una de las afirmaciones del pastoreo más valorada para este grupo de consumidores. No en vano, la relación entre el pastoreo y la prevención de incendios es un atributo muy importante y que preocupa a la población (Varela et al., 2014), considerado una función ecológica medioam-

Tabla IV. Resumen de frecuencias de las ideas de
asociación respecto a la leche de cabra de pastoreo
$(\mathrm{n}=1072)$ (Summary of frequencies of association ideas re-
garding herding goat's milk $(n=1072))$.

\begin{tabular}{|c|c|c|}
\hline Palabra & Número total & Porcentaje (\% \\
\hline Natural & 72 & 6.72 \\
\hline Ecológica & 71 & 6.62 \\
\hline Sabor & 70 & 6.53 \\
\hline Calidad & 65 & 6.06 \\
\hline Sostenibilidad & 57 & 5.32 \\
\hline Salud & 56 & 5.22 \\
\hline Bienestar & 43 & 4.01 \\
\hline Saludable & 41 & 3.82 \\
\hline Sana & 40 & 3.73 \\
\hline Animal & 27 & 2.52 \\
\hline Campo & 24 & 2.24 \\
\hline Fresca & 22 & 2.05 \\
\hline Certificación & 21 & 1.96 \\
\hline Fuerte & 21 & 1.96 \\
\hline Medio & 21 & 1.96 \\
\hline Positiva & 21 & 1.96 \\
\hline Ambiente & 19 & 1.77 \\
\hline Buena & 18 & 1.68 \\
\hline Rural & 18 & 1.68 \\
\hline Cara & 16 & 1.49 \\
\hline Queso & 15 & 1.40 \\
\hline Cabra & 13 & 1.21 \\
\hline Sabrosa & 11 & 1.03 \\
\hline Infancia & 11 & 1.03 \\
\hline Leche & 10 & 0.93 \\
\hline Desconocimiento & 9 & 0.84 \\
\hline Densa & 3 & 0.28 \\
\hline Desagradable & 3 & 0.28 \\
\hline Verde & 3 & 0.28 \\
\hline
\end{tabular}

biental de gran interés en Andalucía (Barba et al., 2016) y que ya ha sido utilizada en campañas publicitarias para el consumo de productos ganaderos pastorales (Interovic, 2019).

La leche, así como sus productos elaborados, procedentes de sistemas pastorales tiene mejores propiedades nutricionales y sensoriales (Álvarez et al., 2007; Morand Fehr et al., 2007). Esto es positivo ya que la aceptación de los consumidores por los alimentos depende en gran medida de las preferencias sensoriales. Además, el consumidor moderno considera los alimentos cada vez más como una parte importante de sus hábitos saludables (Mogendi et al., 2016). Los resultados para la afirmación "La leche de pastoreo tiene una alta calidad sensorial y nutricional" muestran que los consumidores están convencidos de ello, partiendo del Clúster A como aquellos que más están de acuerdo hasta el Clúster $\mathrm{C}$ con menor valoración. Con respecto a la edad, diferentes estudios han demostrado que las personas mayores están generalmente más a favor de los alimentos con beneficios nutricionales. También, las mujeres son más favorables a los alimentos que confieren beneficios nutricionales que sus homólogos masculinos (Mogendi et al., 2016; Kraus et al., 2017).

En cuanto a la dimensión social, el pastoreo puede ser en algunos territorios la única actividad que pueden desarrollar los habitantes de zonas rurales (especialmente en las zonas aisladas y menos favorecidas) y que, por lo tanto, representa una oportunidad de ingresos donde no hay otra posible (Ruiz et al., 2019). Además, contribuye a la estabilidad de la población en zonas gravemente amenazadas por la despoblación y el abandono: una de las líneas de acción más importante de la Unión Europea en lo referente al desarrollo rural (Merino y Prats, 2020). Los consumidores valoran positivamente la afirmación de "La leche de pastoreo favorece la fijación de la población en el medio rural", donde el Clúster A obtiene la mayor puntuación (4.87), el Clúster B (4.40) y el Clúster C la menor (3.77), encontrando diferencias entre todos ellos de forma estadísticamente significativa para un nivel de significación de 0.05 .

Existe una alta biodiversidad de razas caprinas autóctonas a nivel europeo (Galal, 2005), encontrándose éstas adaptadas a diferentes ecosistemas donde son las más eficaces en el aprovechamiento de recursos naturales pastorales. Según los consumidores a la afirmación "La leche de pastoreo implica el uso de razas ganaderas autóctonas", en todos los casos se encuentran por encima de la media, siendo la mayor puntuación en el Clúster A.

La relación del pastoreo con el medioambiente es estrecha, ya que es una actividad que se realiza en diferentes ecosistemas y paisajes y principalmente en aquellos con alto valor ecológico (Maldonado et al., 2019), siendo una de las herramientas que los mantienen y modelan. A la afirmación "La leche de pastoreo promueve la defensa del medio ambiente" los consumidores están muy acuerdo con la afirmación con puntuaciones de 4.87 en el Cluster A, 4.46 en el Cluster B y 3.43 en el C. Diferentes estudios afirman que la conciencia ambiental media positivamente en la intención 
de compra de los productos provenientes del pastoreo (Li et al., 2016), por tanto, estas valoraciones de los tres grupos de consumidores son positivas.

Por último, no existe una definición del término producto procedente del pastoreo en ninguna normativa de rango europeo, tan sólo la legislación sobre ganadería ecológica se refiere a la obligación de que los animales tengan acceso a pasto. Los consumidores han dado la mayor puntuación a la afirmación "La leche de pastoreo conlleva que los animales salgan a campo todos los días" en todos los clusters, a excepción del Clúster A cuya puntuación se asemeja a las dadas a otras afirmaciones.

\section{PALABRAS}

Dentro de los resultados para el análisis de texto, las palabras más frecuentemente asociadas a la leche de cabra de pastoreo por los consumidores han sido clasificadas dentro de la categoría principal de positivas, como por ejemplo "natural", "ecológica", "sabor", "calidad", "sostenibilidad", "salud-saludable-sana" o "bienestar". Estos resultados coinciden con los encontrados en otros estudios, como por ejemplo con consumidores de la región del Tirol comparando leche de vaca procedente de alimentación basada en pastoreo frente a heno, en términos como "orgánica", "bienestar animal", "natural" o "salud" (Busch et al., 2018), o con los hallazgos para consumidores alemanes (Weinrich et al., 2014) comparando sistemas vacunos de producción de leche ( in o con acceso al exterior), con asociación del pastoreo con términos como "respetuoso con los animales", "saludable", "cerca de lo natural".

Aunque con baja frecuencia, también se han encontrado palabras categorizadas como negativas, que pueden estar relacionadas con cierta desconfianza del consumidor, con términos como "desconocimiento" o "rara". A pesar de que actualmente existe normas privadas de certificación de la leche de pastoreo, están principalmente orientadas a aspectos relacionados con el bienestar animal y condiciones de acceso a los pastos (Lundmark et al., 2018); no obstante, existen investigaciones acerca de la posible diferenciación en composición de leches procedentes de pastoreo (Lahlou et al., 2014; O'Callaghan et al., 2018) que con un desarrollo futuro pueden ayudar a crear certificaciones que sean más confiables por los consumidores, en la misma medida que los sistemas alimentarios de la industria láctea puedan ser adaptados sin perjuicio económico, sanitario o merma significativa de la producción, al menos en modelos alimentarios mixtos (Fajardo et al., 2015). También es posible explorar nuevos canales de comercialización para alimentos de origen animal de alto valor, como por ejemplo las redes sociales (Elghannam y Mesías, 2018), que se muestran como una oportunidad de dar a conocer rápidamente e introducir los productos en el mercado, supliendo el desconocimiento que los consumidores declaran tener de ellos.

\section{CONCLUSIÓN}

Europa es una potencial productora de leche de cabra de pastoreo, pudiendo ser una solución para la supervivencia de sistemas ganaderos sostenibles y tradicionales. Es necesario fijar un marco normativo que defina las condiciones a cumplir para poder comercializar bajo la denominación de leche de pastoreo, que actualmente no existe.

Los consumidores asocian la leche de pastoreo con causas motivacionales clave para su comercialización como la salud y/o la sostenibilidad. Relacionan la leche de cabra de pastoreo con atributos positivos de sostenibilidad que actualmente cumplen los sistemas ganaderos pastorales de caprino de leche existentes en Europa. Además, al pensar en la leche de pastoreo, los conceptos que asocian con él son en su mayoría de carácter positivo, ligados a atributos como la salud, el bienestar animal y la calidad sensorial.

Estos conceptos pueden convertirse en factores de compra decisivos para el consumidor, y por tanto deben ser tenidos en cuenta a la hora de introducir y comercializar un nuevo producto como la leche de cabra de pastoreo, siguiendo y ampliando las experiencias ya implementadas por la gran distribución con la leche de vaca.

No obstante, aún son necesarias más investigaciones y futuros estudios con el fin de seguir aportando herramientas de marketing a las empresas del sector lácteo para que puedan diversificar su producción, adaptando sus productos y objetivos a las preferencias actuales de un consumidor cada vez más exigente.

\section{AGRADECIMIENTOS}

El presente trabajo ha sido desarrollado bajo el proyecto TRANSFORMA 2019-2021 “Retos de los sistemas

Tabla V. Categorías y subcategorías de las ideas de asociación de los encuestados con respecto a la leche de cabra de pastoreo $(n=1072)$ (Categories and subcategories of respondents' association ideas regarding herding goat's milk $(n=1072))$.

\begin{tabular}{lccc}
\hline \multicolumn{1}{c}{ Categoría } & $\begin{array}{c}\text { Número } \\
\text { total }\end{array}$ & $\begin{array}{c}\text { De la categoría } \\
\text { principal }(\%)\end{array}$ & $\begin{array}{c}\text { Del total }(\%) \\
(\mathrm{n}=1072)\end{array}$ \\
Positiva & 955 & & 89.09 \\
Salud & 215 & 22.51 & 20.06 \\
$\begin{array}{l}\text { Bienestar animal y } \\
\text { medioambiente }\end{array}$ & 191 & 20.00 & 17.82 \\
Sensorial & 179 & 19.90 & 17.72 \\
Generales & 176 & 17.28 & 15.39 \\
Modelo producción & 98 & 10.26 & 9.14 \\
Certificación & 96 & 10.05 & 8.96 \\
Negativa & 52 & & 4.85 \\
Sensorial & 24 & 46.15 & 2.24 \\
Precio & 16 & 30.77 & 1.49 \\
Salud & 9 & 17.31 & 0.84 \\
Generales & 3 & 5.77 & 0.28 \\
Neutral & 65 & & 6.06 \\
\hline
\end{tabular}


ganaderos andaluces y sus productos (RESGAP)", financiado por Fondo Europeo de Desarrollo Regional, dentro del Programa Operativo FEDER de Andalucía 2014-2020.

Los autores agradecen enormemente la participación de todos/as los consumidores, así como el apoyo y asesoramiento de los compañeros y compañeras del Instituto de Investigación y Formación Agraria y Pesquera y de la Producción Ecológica de la Junta de Andalucía, especialmente del IFAPA Centro Camino de Purchil de Granada y del IFAPA Centro Hinojosa del Duque.

\section{BIBLIOGRAFÍA}

Álvarez, S., Fresno, M., Méndez, P., Castro, N., Fernández, J.R., y Sampelayo, M.S. 2007. Alternatives for improving physical, chemical, and sensory characteristics of goat cheeses: the use of arid-land forages in the diet. Journal of dairy science, vol. 90, no. 5, pp. 2181-2188.

Anderson, J. 2019. Frequent Feedback through Google Forms. PRIMUS, vol. 29, no. 2, pp. 124-137.

Arias-Gómez, J., Villasís-Keever, M.Á., y Miranda-Novales, M.G. 2016. The research protocol III. Study population. Revista Alergia México, vol. 63, no. 2, pp. 201-206.

Arnott, G., Ferris, C.P., y O'connell, N.E. 2017. Welfare of dairy cows in continuously housed and pasture-based production systems. Animal, vol. 11, no. 2, pp. 261-273.

Barba, C., Fernández-Tomillo, L., Jiménez, R., Guzmán, J. R., \& García, A. 2016. Valor ecológico ambiental y conservación de razas ovinas en peligro de extinción en Andalucía. Archivos de zootecnia, vol. 65, no. 251, pp. 445-448.

Bernués, A., Ruiz, R., Olaizola, A., Villalba, D., y Casasús, I. 2011 . Sustainability of pasture-based livestock farming systems in the European Mediterranean context: Synergies and trade-offs. Livestock Science, vol. 139, no. 1-2, pp. 44-57.

Busch, G., Kühl, S., \& Gauly, M. 2018. Consumer expectations regarding hay and pasture-raised milk in South Tyrol. Austrian Journal of Agricultural Economics and Rural Studies, vol. 27, no. 11, pp. 79-86.

Bustillo, Á.G. 2015. El sector lácteo en España. Profesión Veterinaria, vol. 21, no. 84, pp 75-78.

Castel, J.M., Ruiz, F.A., Mena, Y., y Sánchez-Rodríguez, M. 2010. Present situation and future perspectives for goat production systems in Spain. Small Ruminant Research, vol. 89, no. 2-3, pp. 207-210.

Charlton, G.L., y Rutter, S.M. 2017. The behaviour of housed dairy cattle with and without pasture access: A review. Applied Animal Behaviour Science, vol. 192, pp. 2-9.

Cuva, A. 2014. Connecting the dots: a review of traversing the uncharted arena of computer assisted qualitative data analysis software: mapping out QDA miner 4.1 as a first-time user. The Qualitative Report, vol. 19, no. 51, pp. 1-4.

Díaz de Rada, V. 2012. Ventajas e inconvenientes de la encuesta por internet. Papers, vol. 97, no. 1, pp. 193-223.

Diekic, I., Smigic, N., Glavan, R., Miocinovic, J., y Tomasevic, I. 2018. Transportation sustainability index in dairy industry-Fuzzy logic approach. Journal of cleaner production, vol. 180, pp. 107-115.

EUROSTAT. 2019. European statistics. Visto 05 de marzo de 2020, Recuperado el 05 de marzo de 2020, <https://ec.europa.eu/eurostat/ data/database>.

Elghannam, A., y Mesías, F.J. 2018. Las redes sociales como nuevo canal de comercialización de alimentos de origen animal: un estudio cualitativo en España. Archivos de Zootecnia, vol. 67, no. 258, pp. 260-268.

FAOSTAT. 2018. Statistics Division of the Food and Agriculture Organization of the United Nations. Visto 05 de marzo de 2020, <http:// faostat.fao.org>.
Fajardo, M., Mattiauda, D.A., Motta, G., Genro, T.C., Meikle, A., Carriquiry, M., y Chilibroste, P. 2015. Use of mixed rations with different access time to pastureland on productive responses of early lactation Holstein cows. Livestock Science, vol. 181, pp. 51-57.

Galal, S. 2005. Biodiversity in goats. Small Ruminant Research, vol. 60, no. 1-2, pp. 75-81.

Gerini, F., Alfnes, F., y Schiøll, A. 2016. Organic-and animal welfare-labelled eggs: competing for the same consumers? . Journal of Agricultural Economics, vol. 67, no. 2, pp. 471-490.

Gillespiex, J., Nyaupane, N., y McMillin, K. 2013. Producer perceptions of important challenges currently facing the United States meat-goat industry. The Professional Animal Scientist, vol. 29, no. 4, pp. 333-344.

Google. 2019. Create, edit, and format. Alphabet, Mountain View, California, Estados Unidos. Visto 05 de marzo de 2020, <https:// support.google.com/docs/topic/6063584?hl=en\&ref_topic=1360904>.

Grunert, K.G., Hieke, S., y Wills, J. 2014. Sustainability labels on food products: Consumer motivation, understanding and use. Food Policy, vol. 44, pp. 177-189.

Gutiérrez, R. 2018a. Caracterización y valorización de la ganadería pastoral de pequeños rumiantes de razas autóctonas a través del análisis de su sostenibilidad y la calidad de sus productos (Tesis doctoral). Universidad de Sevilla, España.

Gutiérrez, R., Fernández, V.M., Mena, Y., y Delgado, M. 2018b. Fatty acid profile and vitamins $A$ and $E$ contents of milk in goat farms under Mediterranean wood pastures as affected by grazing conditions and seasons. Journal of Food Composition and Analysis, vol. 72, pp. 122-131.

Heerwagen, L.R., Mørkbak, M. R., Denver, S., Sandøe, P., y Christensen, T. 2015. The role of quality labels in market-driven animal welfare. Journal of Agricultural and Environmental Ethics, vol. 28, no. 1, pp. 67-84.

Heise, H., y Theuvsen, L. 2016. What do consumers think about farm animal welfare in modern agriculture? Attitudes and shopping behaviour. International Food and Agribusiness Management Review, vol. 20, n0. 1030-2017-2160, pp. 379-399.

Hennessy, D., Delaby, L., van den Pol-van Dasselaar, A., y Shalloo, L. 2020. Increasing grazing in dairy cow milk production systems in Europe. Sustainability, vol. 12, no. 6, p. 2443.

Ingenbleek, P.T., y Immink, V. M. 2011. Consumer decision-making for animal-friendly products: Synthesis and implications. Animal Welfare, vol. 20 , no. 1, pp. 11-19.

Instituto de Estadística y Cartografía de Andalucía. 2019. Indicadores Andalucía y Provincias. Junta de Andalucía, Sevilla, España. Visto 05 de marzo de 2020, <https://www.juntadeandalucia.es/institutodeestadisticaycartografia>.

Interovic. 2019. Silvopastoreo prescrito para la prevención de incendios; una alternativa viable?. Pau Costa Foundation y Fundación Daniel \& Nina Carasso. Visto 05 de marzo de 2020, <http://www.interovic. es/sites/default/files/04_03_Emma_Sergi_FPC.pdfs.

Joshi, A., Kale, S., Chandel, S., y Pal, D. K. 2015. Likert scale: Explored and explained. British Journal of Applied Science \& Technology, vol. 7, no. 4, p. 396.

Kraus, A., Annunziata, A., y Vecchio, R. 2017. Sociodemographic factors differentiating the consumer and the motivations for functional food consumption. Journal of the American College of Nutrition, vol. 36, no. 2, pp. 116-126.

Kühl, S., Gassler, B., y Spiller, A. 2017. Labeling strategies to overcome the problem of niche markets for sustainable milk products: The example of pasture-raised milk. Journal of dairy science, vol. 100, no. 6, pp. 5082-5096.

Lea, E. y Worsley, T. 2005. Australians' organic food beliefs, demographics and values. British Food Journal, vol. 107, no. 11, pp. 855-869.

Lahlou, M.N., Kanneganti, R., Massingill, L.J., Broderick, G.A., Park, Y., Pariza, M. W., Ferguson, J.D, y Wu, Z. 2014. Grazing increases the concentration of CLA in dairy cow milk. Animal, vol. 8, no 7, pp. 1191-1200. 
Li, X., Jensen, K.L., Clark, C.D., y Lambert, D.M. 2016. Consumer willingness to pay for beef grown using climate friendly production practices. Food Policy, vol. 64, pp. 93-106.

Lundmark, F., Berg, C., y Röcklinsberg, H. 2018. Private animal welfare standards-opportunities and risks. Animals, vol. 8, no. 1, pp. 4.

MAPA (Ministerio de Agricultura, Pesca y Alimentación). 2018. El sector ovino y caprino en cifras. Principales indicadores económicos. 2018. Subdirección General de Productos Ganaderos. Ministerio de Agricultura, Pesca y Alimentación. Gobierno de España. Visto 05 de marzo de 2020, <https://www.mapa.gob.es/es/ganaderia/publicaciones/indicadoreseconomicosovinoycaprinocarne2018_tcm30428265.pdfs.

Madry, W., Mena, Y., Roszkowska, B., Gozdowski, R., Hryniewski, R., y Castel, J.M. 2013. An overview of farming system typology methodologies and its use in the study of pasture-based farming system: a review. Spanish Journal of Agricultural Research, vol. 11, no. 2, pp. 316-326.

Maldonado, A.D., Ramos, D., y Aguilera, P.A. 2019. The Role of Cultural Landscapes in the Delivery of Provisioning Ecosystem Services in Protected Areas. Sustainability, vol. 11, no. 9, p. 2471.

Mena, Y., Gutiérrez, R., Pérez, D., Aguirre, I., Horcada, A. $2014 a$. Proyecto Transhabitat: 037/2013-SEN: "Bases para la elaboración del plan de fomento de la ganadería extensiva ecológica y de la comercialización de sus productos". Informe final. Ed.: Consejería de Agricultura, Pesca y Desarrollo Rural, Junta de Andalucía, Sevilla, España.

Mena, Y., Ruiz, F.A., Gutiérrez, R., Vázquez, M.A., y Castel, J.M. 2014b. La función de la ganadería en el mantenimiento y aprovechamiento de los montes mediterráneos. Ambienta: La revista del Ministerio de Medio Ambiente, vol. 2014, no. 108, pp. 52-67.

Mena, Y., Ruiz, J., Ruiz, F.A., y Castel, J.M. 2016. Characterization and typification of small ruminant farms providing fuelbreak grazing services for wildfire prevention in Andalusia (Spain). Science of the Total Environment, vol. 544, pp. 211-219.

Méndez, C. 2018. Sostenibilidad: La leche fresca de Lidl, la primera que cuenta con certificado de pastoreo y de bienestar animal. Aral, vol. 1649, pp. 44-45

Merino, F., y Prats, M.A. 2020. Why do some areas depopulate? The role of economic factors and local governments. Cities, vol. 97, p. 102506.

Milligan, G.W., yHirtle, S.C. 2012. Clustering and classification methods. Research Methods in Psychology, vol. 2, pp. 189-210.

Mogendi, J. B., De Steur, H., Gellynck, X., y Makokha, A. 2016. Consumer evaluation of food with nutritional benefits: a systematic review and narrative synthesis. International journal of food sciences and nutrition, vol. 67, no. 4, pp. 355-371.

Moosmayer, D.C., y Fuljahn, A. 2010. Consumer perceptions of cause related marketing campaigns. Journal of Consumer Marketing, vol. 27, no. 6, pp. 543-549.

Montejano, S. 2018. Bienestar animal y leche de pastoreo: garantías para el consumidor. AENOR: Revista de la normalización y la certificación, vol. 334, pp. 16-19.

Morand-Fehr, P., Fedele, V., Decandia, M., y Le Frileux, Y. 2007. Influence of farming and feeding systems on composition and quality of goat and sheep milk. Small Ruminant Research, vol. 68, no. 1-2, pp. 20-34.

Nayga, R.M. 1997. Impact of sociodemographic factors on perceived importance of nutrition in food shopping. Journal of Consumer Affairs, vol. 31, no. 1, pp. 1-9.

Newberry, M.G., y lsrael, G.D. 2017. Comparing two web/mail mixedmode contact protocols to a Unimode mail survey. Field Methods, vol. 29, no. 3, pp. 281-298.

Niles, M.T. 2013. 13 Achieving Social Sustainability in Animal Agriculture: Challenges and Opportunities to Reconcile Multiple Sustainability Goals. En Kebreab, E. (Ed.), Sustainable animal agriculture, Davis, California, EEUU, pp. 193-211

Ntassiou, K., y Doukas, I.D. 2019. Recording and mapping traditional transhumance routes in the South-Western Macedonia, Greece. GeoJournal, vol. 84, no. 1, pp. 161-181.
O'Callaghan, T.F., Vázquez, R., Serra, A., Dong, E., Mandal, R., Hennessy, D., McAuliffe, S., Dillon, P., Wishart, D.S., Stanton, C., Ross, R.P. 2018. Pasture Feeding Changes the Bovine Rumen and Milk Metabolome. Metabolites, vol. 8, no. 2, p. 27.

Pazzola, M., Dettori, M. L., y Vacca, G.M. 2017. The Sarda Goat, a Resource for the Extensive Exploitation in the Mediterranean Environment. En Sustainable Goat Production in Adverse Environments: Volume II. Springer, Cham, pp. 181-190.

Pulina, G., Milán, M.J., Lavín, M.P., Theodoridis, A., Morin, E., Capote, J., Thomas, D.L., Francesconi, A.H.D. y Caja, G. 2018. Invited review: Current production trends, farm structures, and economics of the dairy sheep and goat sectors. Journal of dairy science, vol. 10, no. 8, pp. 6715-6729.

Robles, A. B., Ruiz-Mirazo, J., Ramos, M.E., y González, J.L. 2009. Role of livestock grazing in sustainable use, naturalness promotion in naturalization of marginal ecosystems of southeastern Spain (Andalusia). En Agroforestry in Europe. Springer, Dordrecht, pp. 21 1-231.

Rubino, R. 2014. A special section on latte Nobile: An evolving model. Journal of Nutritional Ecology and Food Research, vol. 2, no. 3, pp. 214-222.

Ruiz, F.A., Castel, J.M. y Mena, Y. 2019. Current status, challenges and the way forward for dairy goat production in Europe. Asian-Australasian journal of animal sciences, vol. 32, no. 8, p. 1256

Ruiz, F.A., Mena, Y., Castel, J.M., Guinamard, C., Bossis, N., Caramelle, E., Contu, M., Sitzia, M., y Fois, N. 2009. Dairy goat grazing systems in Mediterranean regions: A comparative analysis in Spain, France and Italy. Small Ruminant Research, vol. 85, no. 1, pp. 42-49.

Scarpato, D., Rotondo, G., Simeone, M., Gómez, A. y Gutiérrez, P. 2017. How can food companies attract the consumer concerned about food safety? A logit model analysis in Spain. British Food Journal, vol. 119, no. 8, pp. 1705-1717.

Schuman, H., y Presser, S. 1996. Questions and answers in attitude surveys: Experiments on question form, wording, and context. Nueva York: Sage Publications.

Sepúlveda, C., Ibrahim, M., Bach, O., y Rodríguez, A. 2013. Desarrollo de lineamientos para la certificación de sistemas sostenibles de producción ganadera. Agroforestería en las Américas, vol. 48, pp. 14-20.

Stampa, E., Schipmann-Schwarze, C., y Hamm, U. 2020. Consumer perceptions, preferences, and behavior regarding pasture-raised livestock products: A review. Food Quality and Preference, vol. 80, no. 103872 .

Testa, F., Iraldo, F., Vaccari, A., y Ferrari, E. 2015. Why eco-labels can be effective marketing tools: Evidence from a study on Italian consumers. Business Strategy and the Environment, vol. 24, no. 4, pp. 252-265.

Thomas, D.R. 2006. A general inductive approach for analyzing qualitative evaluation data. American journal of evaluation, vol. 27, no. 2, pp. 237-246.

Thompson, G.D. 1998. Consumer demand for organic foods: what we know and what we need to know. American Journal of Agricultural Economics, vol. 80, no. 5, pp. 1113-1118.

Valdivielso, I., Bustamante, M.A., Aldezabal, A., Amores, G., Virto, M., Ruiz, J.C., de Renobales, M., y Barron, L.J.R. 2016. Case study of a commercial sheep flock under extensive mountain grazing: Pasture derived lipid compounds in milk and cheese. Food chemistry, vol. 197, no, A, pp. 622-633.

Vanhonacker, F., Verbeke, W., Van Poucke, E., y Tuyttens, F. 2007. Segmentation based on consumers' perceived importance and attitude toward farm animal welfare. International Journal of Sociology of Agriculture and Food, vol. 15, no. 3, pp. 91-107.

Vanhonacker, F., y Verbeke, W. 2014. Public and consumer policies for higher welfare food products: Challenges and opportunities. Journal of Agricultural and Environmental Ethics, vol. 27, no. 1, pp. 153-171.

Van Loo, E.J., Caputo, V., Nayga, R.M., y Verbeke, W. 2014. Consumers' valuation of sustainability labels on meat. Food Policy, vol. 49, pp. 137-150.

Van Riemsdijk, L., Ingenbleek, P., Van Trijp, H., y Van der Veen, G. 2017. Marketing animal-friendly products: Addressing the consumer social 
dilemma with reinforcement positioning strategies. Animals, vol. 7, no. 12, p. 98.

Varela, E., Giergiczny, M., Riera, P., Mahieu, P.A., y Soliño, M. 2014. Social preferences for fuel break management programs in Spain: a choice modelling application to prevention of forest fires. International journal of wildland fire, vol. 23, no. 2, pp. 281-289.

Wei, C.F., Chiang, C.T., Kou, T.C., y Lee, B.C. 2017. Toward sustainable livelihoods: Investigating the drivers of purchase behavior for green products. Business Strategy and the Environment, vol. 26, no. 5, pp. 626-639.

Weinrich, R., Kühl, S., Zühlsdorf, A., y Spiller, A. 2014. Consumer attitudes in Germany towards different dairy housing systems and their implications for the marketing of pasture raised milk. International Food and Agribusiness Management Review, vol. 17, no. 4, pp. 205-222. Willer, H., Schaack, D., y Lernoud, J. 2019. Organic farming and market development in Europe and the European Union. En The World of Organic Agriculture. Statistics and Emerging Trends, Research Institute of Organic Agriculture FiBL and IFOAM-Organics International, pp. 217-254.

Zhang, C., y Conrad, F. 2014. Speeding in web surveys: The tendency to answer very fast and its association with straightlining. Survey Research Methods, vol. 8, no. 2, pp. 127-135. 